\section{Adopting smart meter events as key data for low-voltage network operation}

\author{
Jesús García Prado ${ }^{1}$, Ana González ${ }^{1}$, Sandra Riaño ${ }^{2}$ \\ ${ }^{1}$ Iberdrola, Bilbao, Spain \\ ${ }^{2}$ Tecnalia, Derio, Spain \\ 凶-mail: jgarciapr@iberdrola.es
}

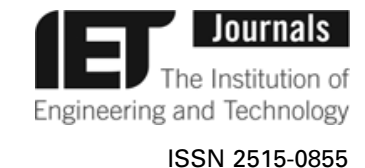

ISSN 2515-0855 doi: 10.1049/oap-cired.2017.0784 www.ietdl.org

\begin{abstract}
A pioneering analysis of smart meter events aimed to enhance low-voltage (LV) network operation by the detection of voltage deviations, repetitive incidents or even outage prevention is presented. The main challenge of using smart meters events is the vast amount of data collected: the average of events gathered in an area per day is around $40 \%$ higher than the number of smart meters installed. To transform this huge quantity of information in network improvements, a set of strategies have been undertaken. The core purpose of this analysis is to establish a more rational and automated processing of smart meter events, aimed to embrace them as key information for network operation.
\end{abstract}

\section{Introduction}

Smart meters are certainly the most relevant devices in the advanced metering infrastructure (AMI) roll-out. The road towards a smart grid passes through them, but AMI deployment is not the last stop. Once the distribution system operators (DSOs) have taken advantage of smart meters to improve the monitoring or billing processes, it is time to go a step forward and to focus on other kinds of data, such as smart meter events.

Events indicate anomalous network situations reported by smart meters, as well as notifications, classified in a broad range of more than 150 types [1].

As can be seen in Table 1, smart meter events cover circumstances related to quality of supply (QoS), demand response, security failures, fraud, communications or specific issues of network devices.

In addition to this classification, smart meter events are prioritised as spontaneous or non-spontaneous. The first ones are reported to the AMI Head End when events take place, while the second ones are stored in the smart meters until there is a request from the AMI Head End to retrieve them to DSO (e.g. once per week).

Although event registering and sending is configurable by smart meter firmware (e.g. types of events identified and transmission period), the quantity of events is really large. Moreover, as the smart meters are still being deployed at the national level [2], the knowledge collected from the present experience is of great importance to foresee the number of events to manage and define final tool capabilities in the coming years.

The analysis presented in this study is based on real low-voltage (LV) network data collected from the Bilbao area in the north of Spain, within the UPGRID project. A set of strategies have been undertaken to manage this huge amount of information. Firstly, some tools based on Visual Basic for Applications (VBA) have been developed to automate event gathering, filtering and sorting processes. Secondly, the study focuses on the specific event types that are strongly related to QoS, such as overvoltage, undervoltage and loss of neutral. Furthermore, additional set-up parameters such as the time frame or network aggregation level (e.g. fuse box (FB) or secondary substation (SS)) are introduced in the analysis. Thirdly, measurements from distribution transformer supervision meters installed in each SS are taken into account to complement the event data analysis, to improve the detection of hot spots in the LV network.

\section{Methodology}

\subsection{Architecture}

The AMI architecture is shown in Fig. 1. The data concentrator acts as a gateway to smart meter data at the SS level, being able to communicate with the DSO Meter Data Management System (MDMS) through different types of communications. The MDMS holds and processes smart meter data. The prevailing tendency, apart from billing purposes, is to use the smart meters to monitor and improve the LV grid $[3,4]$. Within this framework, smart meter events, which are recorded in the MDMS database, can be retrieved to be analysed offline. Moreover, in Fig. 1, LV NMS stands for LV Network Management System. It is a system developed within the UPGRID Spanish demonstrator to operate the LV network similar to the medium voltage (MV) supervisory control and data acquisition (SCADA).

\subsection{Preliminary analysis of events}

As an initial approach, samples of events were extracted from the MDMS to analyse and structure the presentation of results. The network covered is referred to the whole area of Vizcaya, where there are over 500,000 smart meters deployed. First highlights report that an average of 700,000 events are recorded per day in this area, belonging to all the types appearing in Fig. 1. Since the analysis is aimed to improve the LV network management, and given the vast amount of data retrieved, an approach to relate residential end-user claims regarding voltage quality with smart meter events is selected. This results from the attempt to reduce the number of events to a manageable quantity.

\subsection{Cross-matching of claims and events}

Incidents related to QoS reported by customers during 2015 in the selected area have been compared with events registered in MDMS (i.e. overvoltage and undervoltage). This cross-matching is aimed to determine if these kinds of incidents could be detected in advance leading to a better management and QoS. This analysis approach points out that:

- There is misleading information due to the crucial role of human factor in claims reporting. In some cases, the date of the claim is far away from the date of the reported incident. In other cases, the 
Table 1 Event classification according to its source

\begin{tabular}{|c|c|c|c|}
\hline Group & Subgroup & Description & $\begin{array}{c}\text { Types of } \\
\text { events }\end{array}$ \\
\hline \multirow[t]{4}{*}{ standard } & standard & $\begin{array}{c}\text { not recorded as other } \\
\text { category }\end{array}$ & 50 \\
\hline & $\begin{array}{l}\text { contracted } \\
\text { power }\end{array}$ & contract changes & 24 \\
\hline & firmware & firmware changes & 1 \\
\hline & synchronisation & date changes & 1 \\
\hline $\begin{array}{l}\text { cut-off } \\
\text { control }\end{array}$ & - & related to cut-off & 14 \\
\hline \multirow[t]{2}{*}{ Qos } & open incidents & beginning of incidents & 13 \\
\hline & closed incidents & end of incidents & 13 \\
\hline fraud & - & fraud detection & 14 \\
\hline $\begin{array}{l}\text { demand } \\
\text { response }\end{array}$ & - & demand response & 20 \\
\hline $\begin{array}{l}\text { high } \\
\text { occurrence }\end{array}$ & - & communication issues & 6 \\
\hline \multirow[t]{2}{*}{ security } & group one & $\begin{array}{l}\text { successful operations } \\
\text { related to security }\end{array}$ & 6 \\
\hline & group two & $\begin{array}{l}\text { erroneous operations } \\
\text { related to security }\end{array}$ & 7 \\
\hline
\end{tabular}

electrical knowledge of end users is focused on outages, even the scheduled ones.

- Most of the incidents are related to power interruption, even due to AMI roll out scheduled interruptions, and these do not give any clues to improve distribution.

- Event recording can be improved. For example, sometimes smart meters from different manufacturers record different events during the same supply incident. Moreover, in some cases there are meters which are not recording any event since certain date in the MDMS, while in other cases only certain events seems to be missed.

Thus, another approach is needed as the objective of smart meter events analysis is the improvement of the LV network management.

\subsection{Semi-automated analysis}

Next steps at smart meter events analysis must be aimed at the automation of events searching and processing, adding intelligence to the database request. This approach is divided into:

- Specification and implementation of analysis tools.

- Delimit the scope of analysis.

- Testing and improvement of analysis tools.

Although the core analysis is done for Vizcaya, it is decided to add some extra locations in Spain, also covered at previous cross-matching. These locations are Burgos, Castellón and Madrid, which are included with the aim of covering different climates, balancing distribution of rural and urban areas and keeping a large variety of customers. Then, a more representative sample of data is

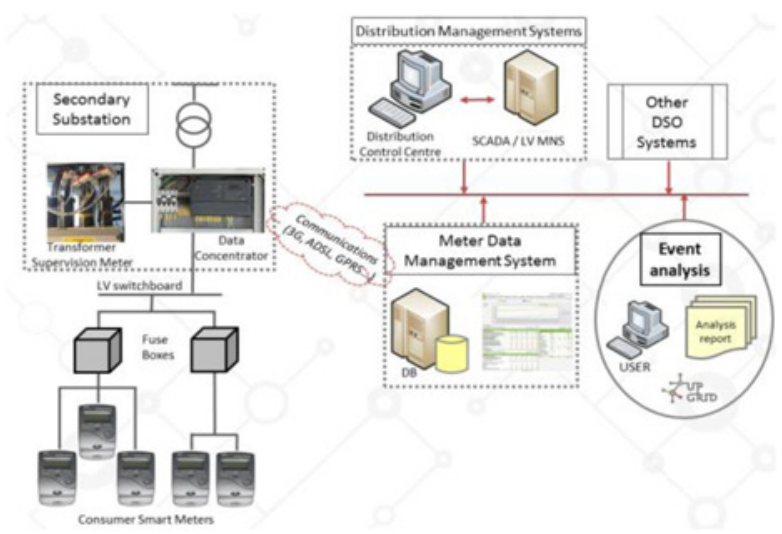

Fig. 1 Offline event analysis within AMI infrastructure available for the study, testing the replicability proof of concept as well.

\subsubsection{Specification and implementation of analysis} tools: Excel VBA is chosen as development environment, due to:

- Accuracy, repeatability and replicability for MDMS database requests.

- Possibility of dumping queries results directly to Excel files, which are persistent and portable files.

- Flexibility and modularity to add new features based on analysis results.

- Ability to group and compare network elements (zones, SSs, FBs etc.).

2.4.2 Delimit the scope of analysis: Due to the early stage of leveraging smart meter events as key data for network operation, the analysis is also an iterative process, as indicated in Fig. 2.

Some types of events have been selected (see Table 2) in order to delimit the scope of the analysis. These events are the ones being helpful (based on Iberdrola Distribución experience) to enhance LV network operation or to detect the potential loss of events record in MDMS.

In spite of this selection, the analysis of loss of neutral events showed that there was a considerable quantity of false positives. These events belong to smart meters without neutral connection due to installation issues, even if the power supply and the billing are correct. For these reasons, they were excluded at this stage.

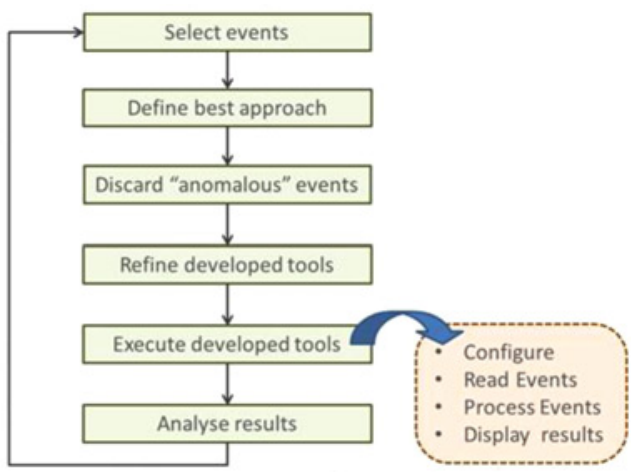

Fig. 2 Events analysis flow chart

Table 2 Events selected for the analysis

\begin{tabular}{|c|c|c|c|}
\hline Type & Group & $\begin{array}{l}\text { Event } \\
\text { Id. }\end{array}$ & Description \\
\hline \multirow[t]{9}{*}{$\begin{array}{l}\text { closed quality } \\
\text { incidents }\end{array}$} & 3 & 13 & $\begin{array}{l}\text { average voltage between phases } \\
\text { is under lower limit }\end{array}$ \\
\hline & 3 & 14 & $\begin{array}{c}\text { phase } 1 \text { voltage is under lower } \\
\text { limit }\end{array}$ \\
\hline & 3 & 15 & $\begin{array}{c}\text { phase } 2 \text { voltage is under lower } \\
\text { limit }\end{array}$ \\
\hline & 3 & 16 & $\begin{array}{c}\text { phase } 3 \text { voltage is under lower } \\
\text { limit }\end{array}$ \\
\hline & 3 & 17 & $\begin{array}{l}\text { average voltage between phases } \\
\text { is over upper limit }\end{array}$ \\
\hline & 3 & 18 & phase 1 voltage is over upper limit \\
\hline & 3 & 19 & phase 2 voltage is over upper limit \\
\hline & 3 & 20 & phase 3 voltage is over upper limit \\
\hline & 3 & 22 & $\begin{array}{c}\text { long-term outage detected at } \\
\text { phase } 1\end{array}$ \\
\hline \multirow[t]{4}{*}{ standard } & 1 & 1 & $\begin{array}{c}\text { meter restart event with missed } \\
\text { data }\end{array}$ \\
\hline & 1 & 2 & $\begin{array}{l}\text { meter restart event without } \\
\text { missed data }\end{array}$ \\
\hline & 1 & 3 & supply failure at meter event \\
\hline & 1 & 7 & loss of neutral \\
\hline
\end{tabular}


For the events analysis, a week period has emerged as the best time frame for aggregating results. From field devices point of view, taking into account a higher level of aggregation than the smart meter has also been revealed as a good approach. This means grouping the analysed events by their connectivity elements, such as FB or SS.

Complementary to the smart meter events analysis, hourly voltage measurements of distribution transformer supervision meters have also been taken into account to detect if the supply incident is located at the SS level.

2.4.3 Testing and improvement of analysis tools: The developed VBA macros are not intended to be either the final end-user tools or integrated applications in the DSO systems at the present time, so they do not have a graphical user interface. The aim is to automate event gathering, filtering and sorting processes, strengthening the accuracy and repeatability of the analysis. The data extracted from MDMS or calculated outcomes are shown in Excel sheets. The accumulated experience of performing analysis will help to select the most useful tool capabilities in order to support a final tool specification in a near future.

The main analysis tools generated are the following VBA Excel macros:

- Get time out of voltage limits of each SS: calculate the time that each SS is out of voltage limits based on the duration of the undervoltage or overvoltage events along a determined period.

- Get time out of voltage limits of each FB: calculate the time that each FB is out of voltage limits based on the duration of the undervoltage/overvoltage events along a determined period.

- Get distribution transformer supervision meter reports: calculate the percentage of hourly measurements that are out of voltage limits. - Search for outage missed events: calculate the percentage of missed events when a specific outage incident takes place.

\section{Results}

\subsection{Detection of missed events}

The aim of this analysis is to quantify how many smart meters that should send an expected event after one known incident really do that (e.g. a power supply interruption affecting all meters fed by one SS).

After an outage, the following events should be recorded in MDMS (see Table 2):

- Supply failure at the meter event (Event 1-3).

- Meter restart event without missed data (Event 1-2) or meter restart event with missed data (Event 1-1).

- Long-term outage detected at phase 1 (Event 3-22). To make the analysis easier, only the outage events at phase 1 are considered, to analyse in the same way the three-phase and monophasic meters.

The conclusion of this study is that around $30 \%$ of events are missed, and that this percentage could be extrapolated to the global recording of events in MDMS. The main cause is that the majority of the events are non-spontaneous, and they are recorded weekly in the MDMS, during a certain timeslot. When this timeslot ends, the smart meter events which have not been sent are not recorded.

Therefore, a new configuration of smart meters is needed in order to discard some events, such as communication events, useful during the roll out, but dispensable after finishing it.

\subsection{Field analysis of events}

The outcomes of undervoltage and overvoltage events analysis during December 2015 in the region/zone of Castellón were delivered some months later to the network maintenance staff, taking advantage of their availability for a field study. This study is aimed to test the truthfulness of the events analysis results.
December 2015 is selected because it has the greatest number of events.

There are two types of connections on the basis of the nominal voltage:

- B01: supplying $133 \mathrm{~V}$, with regulatory voltage limits (seven per cent threshold) between 123 and $142 \mathrm{~V}$ (remaining old LV network). - B02: supplying $231 \mathrm{~V}$, regulatory voltage limits between 215 and $247 \mathrm{~V}$.

After the field analysis of the five worst cases detected which are related to overvoltage, it is verified that there is a one-to-one relationship in $100 \%$ of cases between the distance of FB to SS and time overvoltage limits calculated through events. One of the cases is presented in Fig. 3 (referred to SS 5010000233). The FB with code 984847 , which is the nearest to the SS, is over the voltage limits for a longer time. In contrast, the FB with code 989160 is the one with fewer events and is the farthest from the SS.

Regarding undervoltage events, the 17 worst cases were considered within a field analysis. The conclusion is that there is a one-to-one relationship in $80 \%$ of cases $(15 / 17)$ between distance to SS and time under voltage limits calculated through events. The exceptions are because of connection issues, such as a non-suitable section of connection wires. SS 501000070 is presented in Fig. 4 as an example, being the farthest FB (9958090) the one which has more time below the voltage limit.

So, the conclusion of the field analysis is that the events could offer a valuable input to improve QoS.

\subsection{Wrongly labelled supervision meters}

The results of the transformer supervision meter reports from the Vizcaya network area are presented in the scatter diagrams of Figs. 5 and 6. Here, the list of supervision meters is plotted versus the percentage of hourly measurements out of regulatory ranges during April 2016. It can be seen that the vast amount of almost 5000 supervision meters have no voltage issues, but it is remarkable that there are some of them with $100 \%$ of measurements out of the voltage range in both cases.

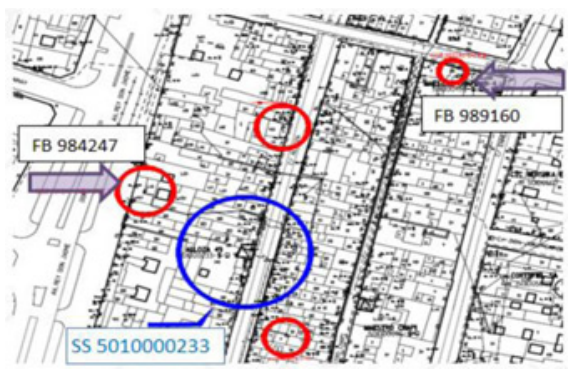

Fig. 3 Field analysis of overvoltage events

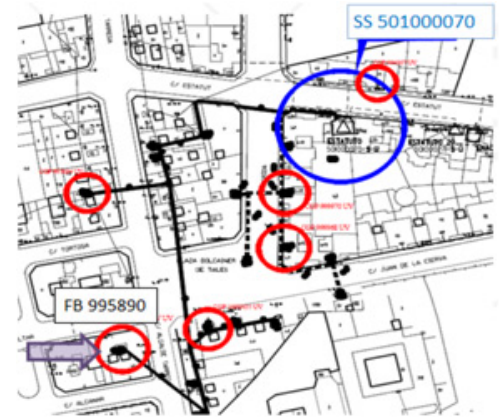

Fig. 4 Field analysis of undervoltage events 


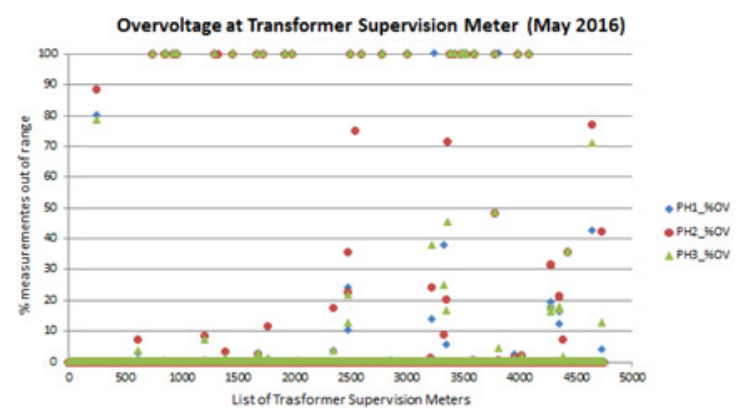

Fig. 5 Overvoltage report results of the transformer supervision meter



Fig. 6 Undervoltage report results of the transformer supervision meter

A deeper analysis indicates that this is caused by an inventory error. The VBA Excel tool which generates the reports calculates the statistics about measurements according to the label of the supervision meter in the database. The calculated report holds the code of the transformer supervisory meter, the service point from where the connection type is assigned, as well as the maximum and minimum voltage measurements, the percentage of measurements over the regulatory voltage read within the period and the average of the measurements for each phase. If a supervision meter is wrongly labelled as B01, its voltage may be within B02 limits, but the tool indicates overvoltage in all the measurements. On the other hand, if a meter is wrongly labelled as B02, its voltage may be within B01 limits, but the tool indicates undervoltage in all the measurements

After checking all the supervision meters from the DSO, 418 out of them have been detected as wrongly labelled. This is an unexpected finding that has led to improve the database.

\subsection{New DSO tool: the virtual register}

Most of the events are non-spontaneous and happened some time ago, so they are analysed in hindsight. A new tool has been developed and implemented to check the real values of voltage

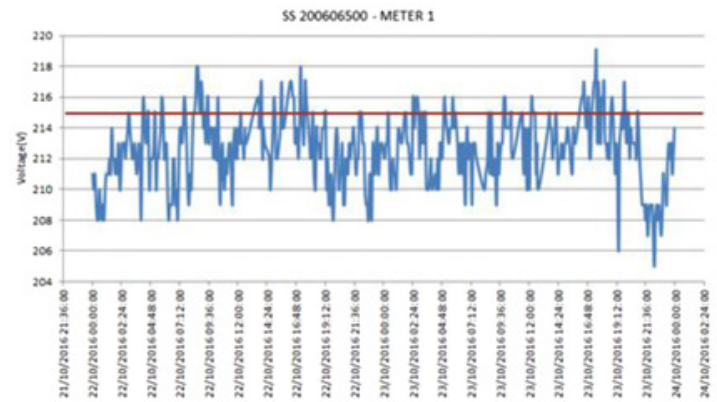

Fig. 7 Most of the meter 1 voltage values (blue line) under regulatory threshold (red line)



Fig. 8 Most of the meter 2 voltage values (blue line) under regulatory threshold (red line)

and current of customer meters: the virtual register. This software tool records voltage and current measurements of specified meters every 5 min for $48 \mathrm{~h}$.

This way the virtual register increases the supervision of the LV grid, providing real measurements from suspicious meters.

\subsection{Detection of weaknesses at supply quality}

After analysing events data from Vizcaya between January 2016 and October 2016, using all the tools mentioned in the study, some issues have emerged.

Overvoltage issues seem to be occasional, which are not clearly detected when measurements of the virtual register are requested.

Conversely, regarding undervoltage events, a set of weaknesses at the LV network have been detected to be reviewed for solution proposal. For example, the FB 3167900, belonging to SS with code 200606500, has two meters connected. These meters are recording undervoltage events for all the weeks of the analysis. After launching the Virtual Register, a supply issue might have been detected, as the voltage measured by both meters is under the voltage limit $(215 \mathrm{~V})$ for almost $48 \mathrm{~h}$ (Figs. 7 and 8 ).

\section{Conclusions}

Although smart meter event information has proven to be promising to network operation, there is still huge improvement potential to take full advantage of it.

On the one hand, the smart meter events offer the DSO the capability of automatically receiving information on LV network incidents. In the light of the performed survey, this information could be valuable to enhance the network operation.

On the other hand, it involves the challenge of dealing with a high amount of data, which should require the application of big data analytics techniques. Therefore the next steps which should be taken are as follows:

- Standardisation of event generation and delivery at customer meters.

- Reduction of the percentage of missed events to ensure the reliability of events analysis.

- Refinement of event processing, according to field performance, to avoid recording false positive events.

- Deep review of events priority to classify them as spontaneous, non-spontaneous or even as non-recording in the MDMS.

- Analysis with even broader time horizon in order to eliminate temporalities or to avoid misunderstanding consumption patterns.

\section{Acknowledgments}

This development is part of the UPGRID project, which has received funding from the European Union's Horizon 2020 research and innovation programme under grant agreement no. 646.531 (http://upgrid.eu/). 


\section{References}

1 'Technical specification of type $5 \mathrm{~m}$ with remote management capabilities and time discrimination', 2011

2 ORDER IET/290/2012, dated 16 February, amending ORDER ITC/3860/2007, dated 28 December, which reviews the electricity rates as of 1 January 2008 in relation to the meter replacement plan. Available at https://www.boe.es/ diario_boe/txt.php?id=BOE-A-2012-2538
3 García, J.: 'Beyond smart meters: management of the LV network'. CIGRE Paris 2016, Preferential Subject 2 - Sub-Topic 3: Impact of developments in energy technology, IT, big data, and further trends in distribution systems, C6-206, 2016

4 Garpetun, L.: 'The challenge of adapting your AMI-system for LV-grid monitoring'. CIRED Conf., Poster session, Theme 3: Grid operation and congestion management, 0244, 2014 